\title{
Comparison of robotic-assisted carotid stenting and manual carotid stenting through the transradial approach
}

\author{
Joshua H. Weinberg, BS, Ahmad Sweid, MD, Kalyan Sajja, MD, M. Reid Gooch, MD, \\ Nabeel Herial, MD, Stavropoula Tjoumakaris, MD, Robert H. Rosenwasser, MD, and \\ Pascal Jabbour, MD
}

Department of Neurological Surgery, Thomas Jefferson University Hospital, Philadelphia, Pennsylvania

OBJECTIVE The objective of this study was to demonstrate the feasibility and safety of CorPath GRX robotic-assisted (RA) transradial (TR) carotid artery stenting (CAS) compared with manual TR CAS.

METHODS The authors conducted a retrospective analysis of a prospectively maintained database and identified 13 consecutive patients who underwent TR CAS from June 2019 through February 2020. Patients were divided into 2 groups: RA (6 patients) and manual (7 patients).

RESULTS Among 6 patients in the RA group with a mean age of $70.0 \pm 7.2$ years, technical success was achieved in all $6(100 \%)$ procedures; there were no technical or access-site complications and no catheter exchanges. Transfemoral conversion was required in $1(16.7 \%)$ case due to a tortuous aortic arch. There were no perioperative complications, including myocardial infarction, stroke, and mortality. The mean procedure duration was significantly longer in the RA group ( $85.0 \pm 14.3$ minutes [95\% Cl 69.9-100.0] vs $61.2 \pm 17.5$ minutes [95\% $\mathrm{Cl} 45.0-77.4], p=0.0231$ ). There was no significant difference in baseline characteristics, fluoroscopy time, contrast dose, radiation exposure, catheter exchanges, technical success, transfemoral conversion, technical or access-site complications, myocardial infarction, stroke, other complications, or mortality.

CONCLUSIONS The authors' results suggest that RA TR CAS is feasible, safe, and effective. Neurovascular-specific engineering and software modifications are needed prior to complete remote control. Remote control has important implications regarding patient access to lifesaving procedures for conditions such as stroke and aneurysm rupture as well as operative precision. Future clinical investigations among larger cohorts are needed to demonstrate reliable performance and patient benefit.

https://thejns.org/doi/abs/10.3171/2020.5.JNS201421

KEYWORDS angioplasty; carotid artery; endovascular; robot; robotic-assisted; stent; transradial; vascular disorders; interventional neurosurgery

I NNOVATIONS in robotic engineering may enhance the consistency and precision of surgical performance. Additionally, robotic advancements may enable operators to perform surgeries from a remote location, inherently increasing patient access to lifesaving procedures as well as limiting occupational hazards such as radiation exposure or infectious disease. ${ }^{1-3}$ To date, robotic surgery systems have been specifically designed for other specialties such as interventional cardiology, thus limiting the use of robotics in neurosurgery. ${ }^{1,2}$ Only recently were robotic surgery systems engineered specifically for neurosurgical procedures enabling robotic-assisted (RA) spine surgeries.

CorPath GRX robotic-assisted platform (Corindus Inc.) was initially designed for coronary interventions. Based on promising clinical trials, its predecessor, CorPath 200 (Corindus Inc.), was approved by the FDA and received $\mathrm{CE}$ marking for percutaneous coronary intervention (PCI). ${ }^{4}$ In this case-control study, we compare RA carotid artery stenting (CAS) using a transradial (TR) approach with manual TR CAS. We aim to assess the feasibility, safety, and efficacy of RA TR CAS compared with manual TR CAS.

\section{Methods}

\section{Study Design}

The study protocol was approved by our institutional review board, and the need for informed consent was

ABBREVIATIONS CAS = carotid artery stenting; $\mathrm{PCI}=$ percutaneous coronary intervention; $\mathrm{RA}=$ robotic-assisted; $\mathrm{TF}=$ transfemoral; $\mathrm{TR}=$ transradial .

SUBMITTED April 22, 2020. ACCEPTED May 18, 2020.

INCLUDE WHEN CITING Published online August 28, 2020; DOI: 10.3171/2020.5.JNS201421. 

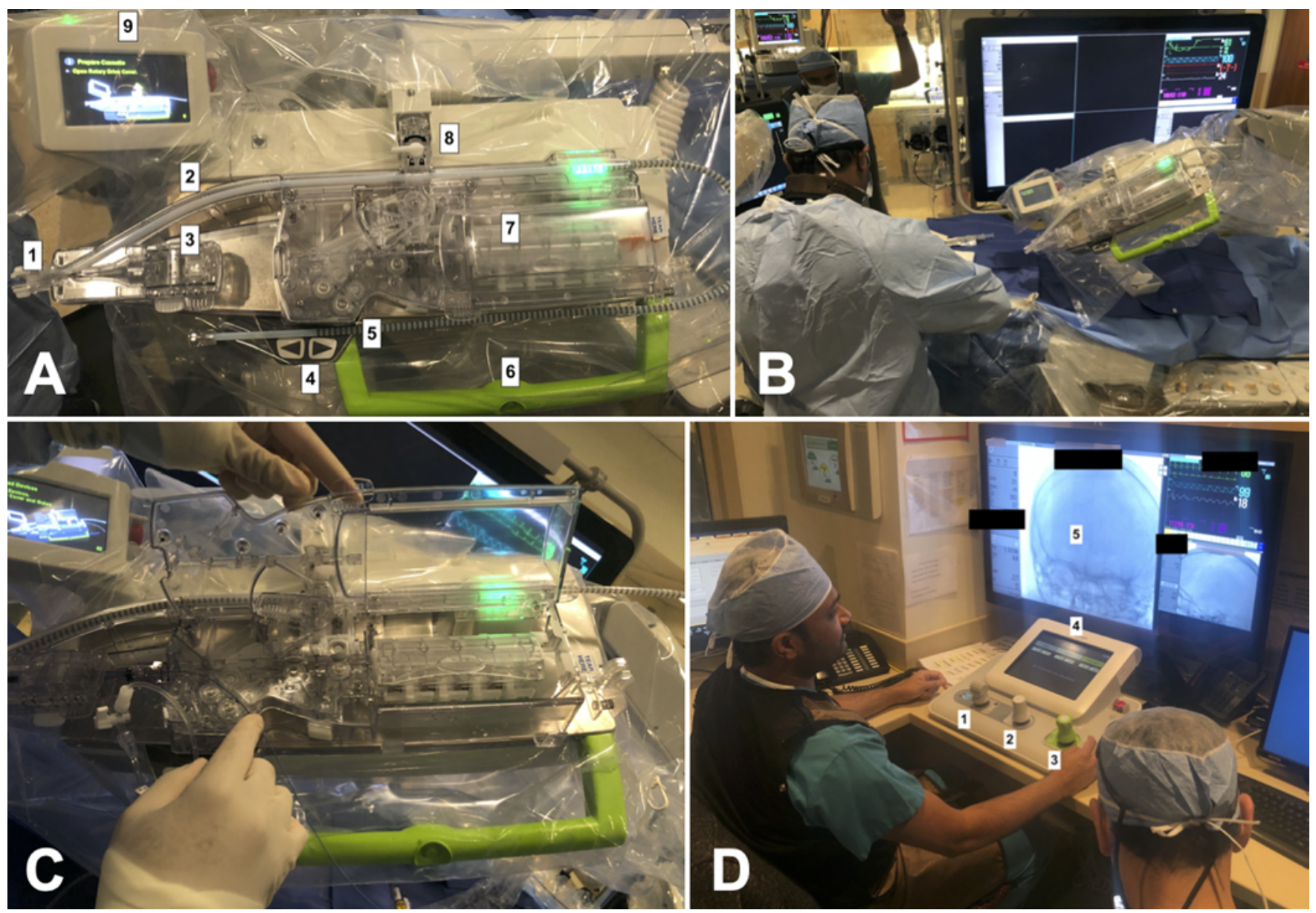

FIG. 1. CorPath CRX RA platform. A: Disposable single-use cassette: 1 , sheath attachment; 2 , guide support track; 3 , guide catheter rotation module; 4 , microadjustment buttons; 5 , device port; 6 , robotic arm toggle button; 7 , guidewire rotation module; 8, cassette lock; and 9, robotic arm feedback console. B: Physician preparing the patient and bedside unit. C: Open cassette with catheter and wire loaded. D: Remote physician unit in radiation-shielded room: 1, device joystick; 2, guidewire joystick; 3, catheter joystick; 4, joystick feedback monitor; and 5, high-definition screen. Figure is available in color online only.

waived due to the study's retrospective nature. We declare that all supporting data are present within the article. We conducted a retrospective analysis of a prospectively maintained database and identified 13 consecutive patients with symptomatic carotid artery stenosis who underwent TR CAS from July 2019 through February 2020. Patients were divided into 2 groups: 6 patients in the RA TR CAS group and 7 patients in the manual TR CAS group (control group). After acquiring the robot, the senior author performed all CAS procedures with robotic assistance. Data collection was performed on baseline demographics, indication for procedure, procedure duration, fluoroscopy time, contrast dose, radiation exposure, catheter exchanges, transfemoral (TF) conversion, technical and access site complications, technical success, perioperative outcomes, and procedure-related complications. TF conversion was defined as failure to complete the procedure after successful cannulation of the radial artery. Technical success was defined as successful stent deployment using robotic assistance. The perioperative period was defined as up to 2 weeks post-CAS, which was the time of the follow-up office visit. We accessed patients' electronic medical records and collected relevant data related to their procedure for data analysis.

\section{Outcomes}

Our primary goal was completion of the endovascular procedure using robotic assistance. We set a safety and efficacy goal to use a single catheter and a single attempt with robotic assistance. If catheterization of the vessel of interest failed with the first catheter, we did not use robotic assistance for the second attempt but instead moved to a manual procedure.

\section{Setup and Technique-RA TR CAS}

The CorPath GRX RA platform includes a bedside and remote physician unit (Fig. 1). The bedside unit includes a single-use disposable cassette, a robotic drive, and an articulated arm. Joysticks on the remote physician unit are used to manipulate devices by translating real-time commands to the cassette (mechanical transmission module). The operator may advance, retract, and rotate catheters and wires in increments as small as $1 \mathrm{~mm}$. The robotic 
system is an open-architecture system compatible with 0.014- and 0.018-inch guidewires, rapid exchange catheter systems, and other devices.

The remote physician unit was stationed outside the room, eliminating physician radiation exposure and minimizing technician exposure. The remote unit includes fluoroscopy and hemodynamic monitors to enable realtime visualization. Axial and rotational movements are performed using joysticks on the remote physician unit capable of translating precise and highly sensitive movements, enabling precise navigation. After connecting a power injector to the diagnostic catheter injection port and placing a wireless fluoroscopy pedal outside the room, diagnostic cervical and cerebral angiograms (including 3D angiograms) could be obtained by selecting the proximal vessels.

The robotic arm was prepared under sterile conditions. The disposable cassette was attached to the arm platform, and the proximal end of the catheter intended for use was attached to a hemostatic valve (Copilot). The catheter was secured by the guide slot of the cassette with the copilot attached, and the guide support track was then pulled over the Sim Select catheter (Penumbra) or Benchmark catheter (Penumbra) and locked onto the side port of the radial sheath. Approximately 20 seconds was required to install a device into the cassette. The cassette was then locked and ready for robotic catheter manipulation.

The right radial artery was accessed using a micropuncture needle. This was exchanged for a 6-Fr radial Prelude (Merit) sheath over a 0.021-inch wire. Heparin was administered according to protocol. A coaxial system comprising a 6-Fr Benchmark guide catheter and 5-Fr diagnostic catheters (Penumbra Sim Select or Berenstein Select) over a 0.038 -inch Glidewire (Terumo Medical Inc.) was used. A Benchmark catheter was advanced over a Sim Select catheter and positioned in the aortic arch manually. The catheter was then connected to the bedside unit of the robot, and the physician at the remote unit took over the procedure. The Sim Select was shaped in the arch via robotic assistance, the proximal vessel was then selected, and the Benchmark catheter was advanced manually. A Synchro2 wire (Stryker Neurovascular) was advanced under biplane roadmap via robotic assistance while precisely avoiding the plaque. Using robotic assistance, a Spider FX filter device (Medtronic) was advanced over the wire and deployed in the infrapetrous carotid artery. An Aviator plus balloon (Cordis) was then advanced over the wire, crossing the lesion via robotic assistance, followed by manual balloon angioplasty. The balloon was deflated manually and then removed via robotic assistance. A Precise Pro carotid stent (Cordis) was advanced crossing the lesion via robotic assistance and was then deployed manually. RA removal of the stent catheter was performed, followed by carotid injection and RA removal of the Benchmark catheter. The sheath was then removed with the help of a TR Band (Terumo Medical Inc.).

\section{Case Illustration}

A 62-year-old female with a history of hypertension, aortofemoral bypass, and $\mathrm{a} \geq 40$-pack/year smoking history presented with confusion and dizziness. Head and neck
CTA revealed complete right carotid artery occlusion with reconstitution of the cavernous segment and severe left internal carotid artery stenosis at the carotid bifurcation with plaque. MRI showed acute left cerebral watershed infarcts in the frontal, parietal, and occipital lobes. The patient was managed with subcutaneous heparin and loaded with aspirin and clopidogrel in preparation for RA TR CAS (Fig. 2 and Video 1).

VIDEO 1. RA transradial carotid artery stent placement on digital subtraction angiography. Copyright Pascal Jabbour. Published with permission. Click here to view.

\section{Statistical Analysis}

Analysis was performed using an unpaired t-test, chisquare test, Fisher's exact test, and ANOVA, as appropriate. Data are presented as the mean and 95\% confidence interval (CI) for continuous variables, and as a frequency for categorical variables; $\mathrm{p}<0.05$ was considered statistically significant. All statistical analyses were performed using a statistical package (Stata SE 14.2, StataCorp).

\section{Results}

\section{Procedural and Clinical Outcomes}

Our retrospective case-control study included 13 consecutive patients with symptomatic carotid artery stenosis who underwent TR CAS from June 2019 through February 2020: 6 patients in the RA TR CAS group and 7 patients in the manual TR CAS group (control group) (Table 1).

Among 6 patients in the RA TR CAS group with a mean age of $70.0 \pm 7.2$ years, $1(16.7 \%)$ was female and $5(83.3 \%)$ had left-sided stenosis. The mean procedure duration was $85.0 \pm 14.3$ minutes, the mean fluoroscopy time was $30.5 \pm 7.2$ minutes, the mean contrast dose was $107.0 \pm 29.1 \mathrm{ml}$, and the mean radiation exposure was 98.7 $\pm 49.9 \mathrm{~Gy} \cdot \mathrm{cm}^{2}$. Procedure duration and fluoroscopy time started to reduce after procedure 2, with the exception of procedure 5, which required TF conversion (Fig. 3). Technical success was achieved in all $6(100 \%)$ procedures via RA CAS. There were no cases of technical or access-site complications, and no cases required catheter exchange. TF conversion was required in $1(16.7 \%)$ case due to a tortuous aortic arch. There were no perioperative complications, including myocardial infarction, stroke, or mortality.

Among 7 patients in the manual TR CAS group (control group) with a mean age of $72.0 \pm 7.9$ years, $1(14.3 \%)$ was female and $5(71.4 \%)$ had left-sided stenosis. The mean procedure duration was $61.2 \pm 17.5$ minutes, the mean fluoroscopy time was $24.3 \pm 8.4$ minutes, the mean contrast dose was $85.7 \pm 33.6 \mathrm{ml}$, and the mean radiation exposure was $94.9 \pm 34.4 \mathrm{~Gy} \cdot \mathrm{cm}^{2}$. Technical success was achieved in all 7 (100\%) procedures via manual CAS. There were no cases of technical or access-site complications, and no cases required catheter exchange. TF conversion was required in $2(28.6 \%)$ cases: one due to tortuosity and the other due to a $360^{\circ}$ radial artery loop. There were no cases of perioperative myocardial infarction, stroke, or mortality.

A comparative analysis was performed between the RA TR CAS group and the control group. Procedure time 

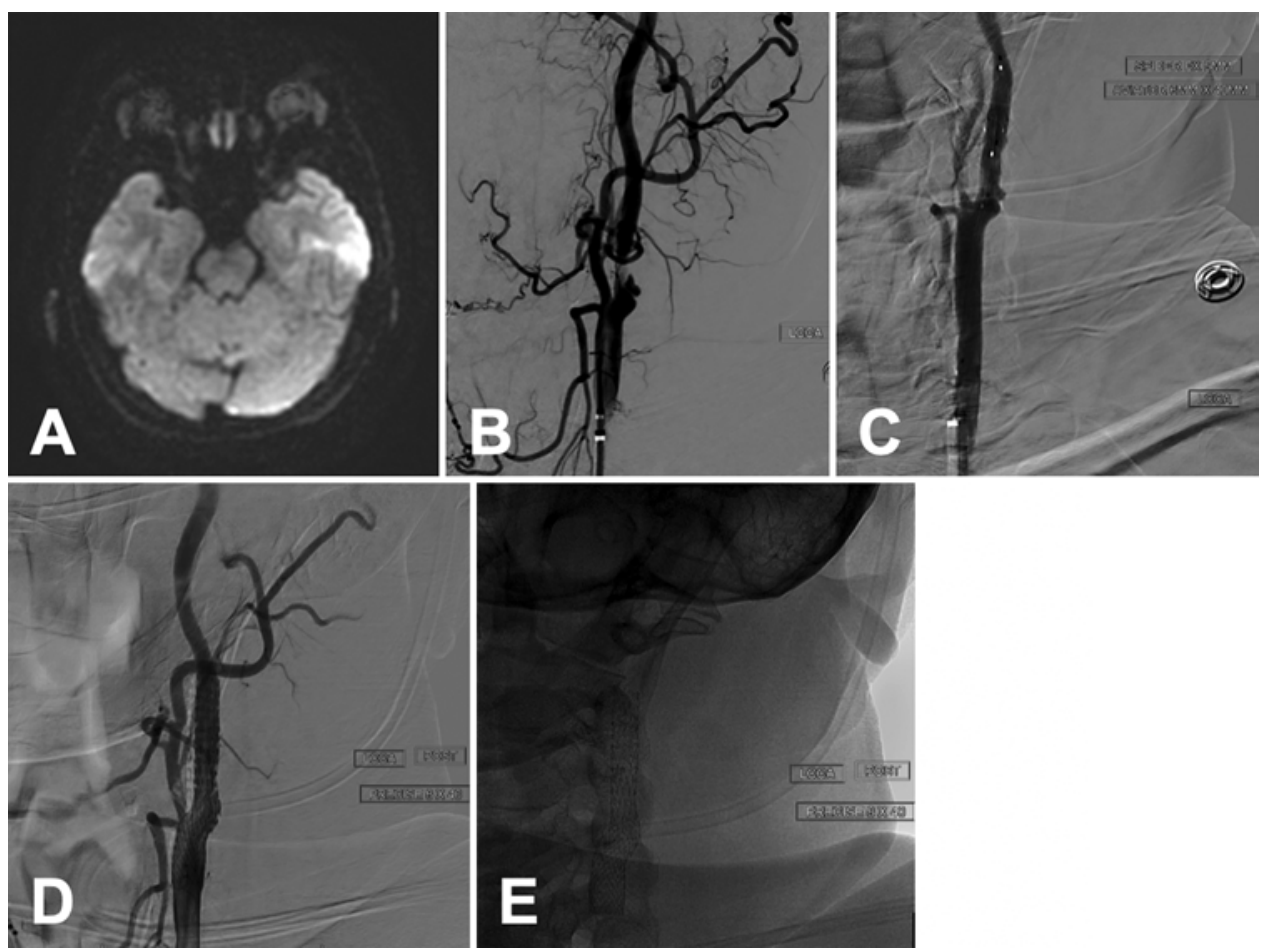

FIG. 2. A: Axial diffusion-weighted MR image showing restricted diffusion in the left middle cerebral artery territory. B: Digital subtraction angiogram obtained prior to intervention showing severe internal carotid artery (ICA) stenosis. C: Balloon angioplasty of the left ICA. D: Resolution of ICA stenosis post-stent placement. E: Left ICA stent on a native image.

was significantly higher in the RA group $(85.0 \pm 14.3$ minutes [95\% CI 69.9-100.0] vs $61.2 \pm 17.5$ minutes [95\% CI 45.0-77.4 minutes], $\mathrm{p}=0.0231$ ). There was no significant difference in baseline characteristics, fluoroscopy time ( $\mathrm{p}$ $=0.1862)$, contrast dose $(\mathrm{p}=0.2804)$, radiation exposure $(\mathrm{p}$ $=0.8758)$, catheter exchanges $(p>0.99)$, technical success $(p>0.99)$, TF conversion ( $>0.99)$, technical $(p>0.99)$ or access-site complications ( $p>0.99)$, myocardial infarction ( $p>0.99)$, stroke $(p>0.99)$, other complications $(p>0.99)$, or mortality $(\mathrm{p}>0.99)$.

\section{Discussion}

\section{Findings Summary}

In the present case-control study, we aimed to assess the feasibility, safety, and efficacy of RA TR CAS compared with manual TR CAS. Technical success was achieved in all $6(100 \%)$ procedures via RA CAS. Procedure duration and fluoroscopy time started to improve after procedure 2 , with the exception of procedure 5 , which required TF conversion. There were no cases of technical or access-site complications, and no cases required catheter exchange. TF conversion was required in $1(16.7 \%)$ case due to a tortuous aortic arch. There were no perioperative complications, including myocardial infarction, stroke, or mortality. The procedure time was significantly longer in the RA group ( $p=0.0231)$; however, there was no significant difference in fluoroscopy time $(\mathrm{p}=0.1862)$, contrast dose $(\mathrm{p}=0.2804)$, or radiation exposure $(\mathrm{p}=0.8758)$. There was also no significant difference in catheter exchanges $(>0.99)$, technical success $(\mathrm{p}>0.99)$, TF conversion $(\mathrm{p}>$
$0.99)$, technical ( $\mathrm{p}>0.99)$ or access-site complications ( $\mathrm{p}$ $>0.99)$, myocardial infarction ( $p>0.99)$, stroke $(p>0.99)$, other complications $(p>0.99)$, or mortality $(p>0.99)$. Our results suggest that RA TR CAS is feasible, safe, and effective. However, our sample size is too small to draw any conclusions.

\section{Current Literature}

In a 2006 clinical trial, Beyar et al. ${ }^{5}$ reported the first use of a remote-control robotic system for endovascular intervention showing that RA PCI is safe and feasible for the treatment of coronary stenosis. Additionally, prospective studies, including the PRECISE trial, have found that the safety and efficacy of RA PCI is comparable to manual PCI. ${ }^{6,7}$ Engineering modifications have expanded the use of robotic systems into neurosurgery, including spine surgery, ${ }^{8}$ epilepsy evaluation, ${ }^{9}$ subcortical surgery, ${ }^{10}$ and endovascular procedures. ${ }^{1-3}$ In 2020 , the first RA neuroendovascular procedures were reported. Sajja et al. ${ }^{1}$ and Nogueira et al. ${ }^{2}$ demonstrated the feasibility of RA CAS, while Mendes Pereira et al. ${ }^{3}$ demonstrated the feasibility of RA stent coiling of a basilar artery aneurysm in Canada. In the present study, we report the first comparison of RA TR CAS and manual TR CAS.

\section{Advantages}

The integration of robotics in endovascular procedures unlocks a new potential for improvements in procedural safety and efficacy. Robotic systems provide enhanced navigation control. ${ }^{1}$ The steady arms and mechanical sen- 
TABLE 1. Comparison of RA versus manual TR CAS

\begin{tabular}{|c|c|c|c|}
\hline Demographics & RA TR CAS & Manual TR CAS & $\mathrm{p}$ Value \\
\hline Mean age, yrs & $70.0 \pm 7.2(62.5-77.5)$ & $72.0 \pm 7.9(64.7-79.3)$ & 0.6456 \\
\hline Female gender & $1(16.7)$ & $1(14.3)$ & $>0.99$ \\
\hline Smoking & $3(50)$ & $3(42.9)$ & $>0.99$ \\
\hline Hypertension & $4(66.7)$ & $7(100)$ & 0.1923 \\
\hline Hyperlipidemia & $6(100)$ & $7(100)$ & $>0.99$ \\
\hline Diabetes mellitus & $1(16.7)$ & $2(28.6)$ & $>0.99$ \\
\hline Symptomatic stenosis & $6(100)$ & $7(100)$ & $>0.99$ \\
\hline Lt-sided stenosis & $5(83.3)$ & $5(71.4)$ & $>0.99$ \\
\hline \multicolumn{4}{|l|}{ Procedure details } \\
\hline Mean procedure time, mins & $85.0 \pm 14.3(69.9-100.0)$ & $61.2 \pm 17.5(45.0-77.4)$ & $0.0231^{*}$ \\
\hline Mean fluoroscopy time, mins & $30.5 \pm 7.2(22.9-38.0)$ & $24.3 \pm 8.4(16.5-32.1)$ & 0.1862 \\
\hline Mean contrast dose, $\mathrm{ml}$ & $107.0 \pm 29.1(70.9-143.1)$ & $85.7 \pm 33.6(54.6-116.8)$ & 0.2804 \\
\hline $\begin{array}{l}\text { Mean radiation exposure, } \\
\mathrm{Gy} \cdot \mathrm{cm}^{2}\end{array}$ & $98.7 \pm 49.9(46.3-151.1)$ & $94.9 \pm 34.4(63.1-126.8)$ & 0.8758 \\
\hline Technical success & $6(100)$ & $7(100)$ & $>0.99$ \\
\hline Catheter exchanges & $0(0)$ & $0(0)$ & $>0.99$ \\
\hline TF conversion & $1(16.7)$ & $2(28.6)$ & $>0.99$ \\
\hline Technical complications & $0(0)$ & $0(0)$ & $>0.99$ \\
\hline Access-site complications & $0(0)$ & $0(0)$ & $>0.99$ \\
\hline Periop outcomes & & & $>0.99$ \\
\hline Myocardial infarction & $0(0)$ & $0(0)$ & $>0.99$ \\
\hline Stroke & $0(0)$ & $0(0)$ & $>0.99$ \\
\hline Other complications & $0(0)$ & $1(14.3)$ & $>0.99$ \\
\hline Mortality & $0(0)$ & $0(0)$ & $>0.99$ \\
\hline
\end{tabular}

Values represent the number of patients (\%) unless stated otherwise. Mean values are presented as the mean \pm SD $(95 \% \mathrm{Cl})$.

* Significant value.

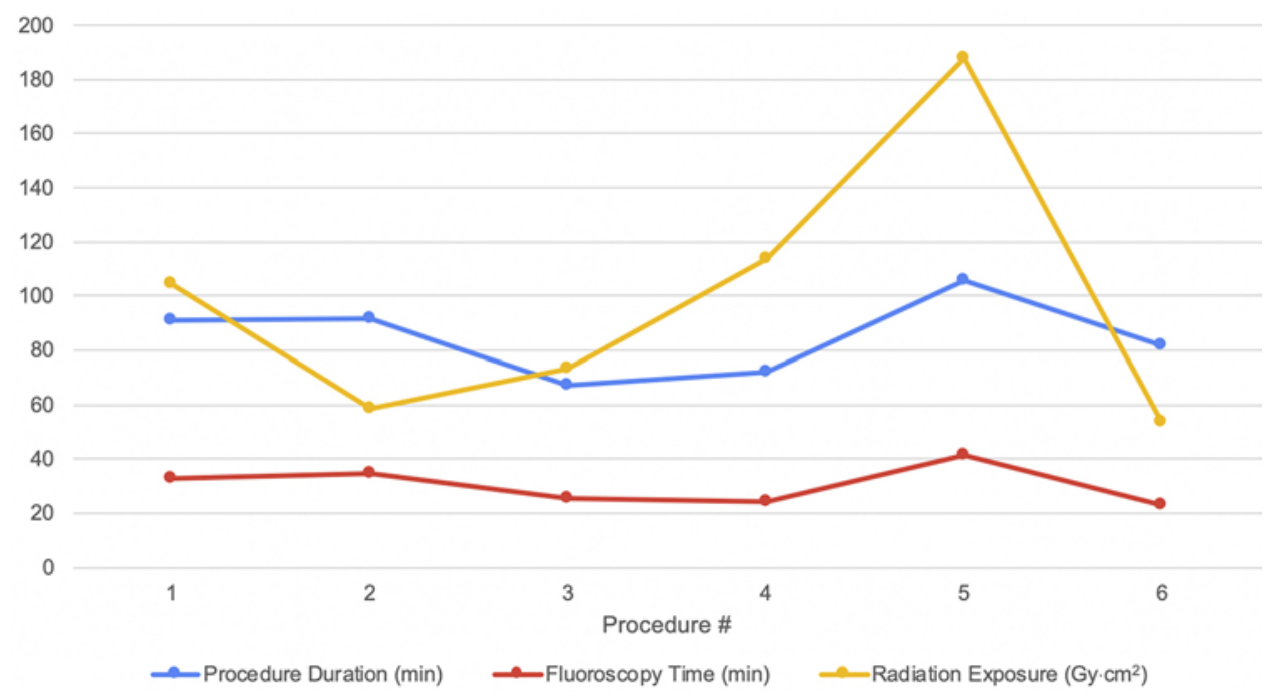

FIG. 3. Procedure metrics for the RA group plotted chronologically by case. Figure is available in color online only. 
sitivity of robotic systems enhance movement accuracy that is unattainable by human hands, regardless of skill or experience. Artificial intelligence algorithms also enable the robotic system to continuously learn and increase consistency among operators. ${ }^{2}$ Robotic systems are particularly advantageous in the setting of tortuous vascular anatomies. ${ }^{1,3}$ The improved straight vector force translation to the guide catheter enhances navigation and prevents herniation into the arch, especially in right $\mathrm{TR}$ procedures requiring left carotid artery access. ${ }^{1}$

The aforementioned robotic enhancements offer obvious benefits to the operator, yet also have the potential to significantly improve patient outcomes. Device manipulation with millimeter precision could reduce technical complications such as stent misplacement, ${ }^{11}$ thereby reducing the need for rescue treatment or re-treatment and the incidence of other vascular complications. Reduction of operator fatigue during prolonged procedures may also improve technical precision of catheter and device positioning and minimize contrast injection, which may reduce fluoroscopy time. ${ }^{12,13}$ Contrast dose reductions are associated with a decreased incidence of contrast-induced nephropathy, and therefore morbidity and mortality. ${ }^{14}$ In the present study, there was no significant difference in patient outcomes or procedure-related metrics other than procedure duration, which is consistent with interventional cardiology literature. ${ }^{7}$ Further studies are needed to determine the benefit to patients of robotic assistance and what adjustments can be made to address unmet clinical needs.

Robotic technology offers the potential to perform emergency endovascular procedures from distant locations, increasing patient access to lifesaving procedures in remote geographic areas. ${ }^{1-3,6,11,15,16}$ In 2019, Patel et al. ${ }^{17}$ reported the successful completion of long-distance teleRA PCIs from 20 miles away, while Madder et al. ${ }^{18}$ was successful from greater than 100 miles away. One of the greatest challenges stroke patients face is timely access to effective reperfusion therapy. ${ }^{19}$ The ability to perform tele-RA mechanical thrombectomy has the potential to decrease time to reperfusion and save countless lives in locations that are not close to thrombectomy-capable or comprehensive stroke centers..$^{1-3}$ If successfully implemented, robotic innovations have the potential to improve patient outcomes and revolutionize stroke care on a global scale. ${ }^{19}$

Robotic systems also enhance the safety and experience of operators..$^{1,2,4}$ As the types of endovascular procedures and procedural volume increase, there is additional concern regarding cumulative radiation exposure. ${ }^{17}$ This may be particularly true of TR procedures, which might be associated with higher radiation exposure as the operator is closer to the radiation source than in TF procedures. ${ }^{1,20}$ RA endovascular procedures reduce exposure to scatter radiation, ${ }^{1,2,6}$ thereby reducing the risk of left-sided brain tumors, melanoma, breast cancer, lens opacities, and accelerated atherosclerosis. ${ }^{21-25}$ Additionally, standing for prolonged durations while wearing heavy radiation protection equipment may lead to orthopedic injuries, most commonly cervical and lumbar injuries or "interventionalist disc disease." ",26,27 Spine conditions have also been shown to correlate with annual case load and number of years in practice. ${ }^{28} \mathrm{RA}$ endovascular procedures may re- duce physical demands that lead to orthopedic injury, reduce operator fatigue due to suboptimal ergonomics, and minimize back pain associated with lead apron use..$^{1,4,29}$ The improved operator ergonomics may also reduce errors due to operator fatigue during prolonged procedures. ${ }^{1}$

\section{Disadvantages}

Although the CorPath robotic system was used in Canada to assist in stent-assisted coiling of a basilar aneurysm, ${ }^{3}$ the CorPath robot and other robotic systems have not yet received FDA approval for intracranial procedures. Many technical nuances may need to be addressed prior to acquiring FDA approval and expanding the role of RA endovascular procedures.

The robotic cassette is not specifically engineered for neuroendovascular procedures, which limits the use of several endovascular devices. More substantial wires, including the 0.035- and 0.038-inch Glidewires, are not compatible with the current cassette design. In the setting of a bovine arch, navigation into the left common carotid artery is limited, as the lack of support provided by compatible devices may result in catheter herniation into the arch. However, Glidewire placement within the manual port enables manual super selection of the supra-aortic vessels and continued use of the robotic system to access other supra-aortic vessels. ${ }^{1}$ As a result, operator presence is required to manually perform the following: vascular access, catheter advancement into the arch, and device deployment. The operator or a member of the supporting staff must also be present when changes in table position are needed.

The lack of cassette capability to manipulate microcatheters and other over-wire coaxial systems significantly limits robotic use for intracranial procedures and with most neuroendovascular devices. ${ }^{1,2}$ However, recent modifications in neuroendovascular-specific engineering (cassette) and software (active device fixation control) to the CorPath GRX Robotic System enable automated guidewire manipulation relative to the microcatheter via a cassette compatible with neuroendovascular-specific instruments. In a live porcine model with simulated neuroendovascular pathology, the robotic cassette successfully and safely manipulated neurovascular-specific guidewires and microcatheters, demonstrating navigation enhancements more suited for a delicate and tortuous neurovasculature. ${ }^{30}$ In another study using flow and porcine models with simulated neuroendovascular pathology, Britz et al. ${ }^{31}$ also demonstrated the feasibility of RA intracranial neurointervention via successful navigation and deployment of small-gauge devices such as stents and coils. These advancements and success in preclinical models represent an important step toward the development of a neurovascularspecific robotic system.

The lack of reliable haptic feedback compared with manual procedures is also a significant concern, particularly for intracranial procedures involving delicate or tortuous anatomies. ${ }^{1,2,19}$ During the first-in-human neurointervention in Canada, Mendes Pereira et al. ${ }^{3}$ found that observation of subtle shape and motion changes on the console screen sufficiently compensated for altered sensory feedback. However, this may not always be the case. 
Technology enabling haptic feedback with vascular robotic systems remains an engineering challenge, ${ }^{32,33}$ which may need to be addressed prior to widespread adoption of a neurovascular-specific robotic system. ${ }^{1}$

Other potential limitations include costs of the robotic system and disposable cassettes, internet connection speeds from remote locations possibly causing delay, inability for manual rescue treatment, and the learning curve. ${ }^{1}$ Of note, Weisz et al. ${ }^{6}$ demonstrated that the learning curve for the CorPath 200 robotic system is short, and Sajja et al. ${ }^{1}$ found that performance measures such as procedure time improved after 3 RA procedures without compromising patient safety. In the present study, procedure time improved with additional cases, with the exception of a tortuous arch that required TF conversion.

\section{Preliminary Results and Future Directions}

Our results suggest that RA TR CAS is feasible, safe, and effective with additional benefits of reduced occupational radiation exposure and orthopedic injuries associated with lead aprons and high patient volume. ${ }^{1}$ However, RA CAS had a significantly longer procedure time and critical portions of the procedure, such as stent deployment and bovine arch catheterization, may still need to be performed manually. ${ }^{19}$ Additional engineering and software modifications are needed prior to complete remote control and widespread adoption by the endovascular community. Wireless control of table position, fluoroscopy pedals, and power injectors as well as steerable microcatheters may be important steps toward complete remote control, expanding the role of robotic systems in neurosurgery and eliminating radiation exposure to the entire healthcare team. ${ }^{1}$

The major future implication of RA neuroendovascular procedures may be timely access to emergency procedures for conditions such as aneurysm rupture or stroke in locations distant from thrombectomy-capable stroke centers. ${ }^{1-3,19}$ In preclinical models, Britz et al. ${ }^{31}$ demonstrated the feasibility of intracranial neurointervention using the CorPath GRX robotic system as well as improved navigation and manipulation of neuroendovascular-specific devices enabled by recent neuroendovascular-specific engineering and software modifications. ${ }^{30}$ In addition to further engineering innovations, future prospective clinical trials among larger cohorts are needed to demonstrate reliable control, safety, and efficacy. ${ }^{19}$

\section{Study Limitations}

The limitations of this study include the small sample size, lack of long-term patient follow-up, and lack of a randomized controlled design. This was a single-center study of TR CAS performed with robotic assistance or manually by neurointerventionalists; therefore, our data may lack generalization.

\section{Conclusions}

Our results suggest that RA TR CAS is feasible, safe, and effective. Additional neurovascular-specific engineering and software modifications are needed prior to complete remote control and widespread adoption. Complete remote control has important implications regarding patient access to lifesaving procedures for conditions such as stroke and aneurysm rupture as well as operative precision. Future clinical investigations among larger cohorts are needed to demonstrate reliable performance and patient benefit.

\section{References}

1. Sajja KC, Sweid A, Al Saiegh F, et al. Endovascular robotic: feasibility and proof of principle for diagnostic cerebral angiography and carotid artery stenting. J Neurointerv Surg. 2020;12(4):345-349.

2. Nogueira RG, Sachdeva R, Al-Bayati AR, et al. Robotic assisted carotid artery stenting for the treatment of symptomatic carotid disease: technical feasibility and preliminary results. J Neurointerv Surg. 2020;12(4):341-344.

3. Mendes Pereira V, Cancelliere NM, Nicholson P, et al. Firstin-human, robotic-assisted neuroendovascular intervention. $J$ Neurointerv Surg. 2020;12(4):338-340.

4. Maor E, Eleid MF, Gulati R, et al. Current and future use of robotic devices to perform percutaneous coronary interventions: a review. J Am Heart Assoc. 2017;6(7):e006239.

5. Beyar R, Gruberg L, Deleanu D, et al. Remote-control percutaneous coronary interventions: concept, validation, and firstin-humans pilot clinical trial. J Am Coll Cardiol. 2006;47(2): 296-300.

6. Weisz G, Metzger DC, Caputo RP, et al. Safety and feasibility of robotic percutaneous coronary intervention: PRECISE (Percutaneous Robotically-Enhanced Coronary Intervention) Study. J Am Coll Cardiol. 2013;61(15):1596-1600.

7. Mahmud E, Naghi J, Ang L, et al. Demonstration of the safety and feasibility of robotically assisted percutaneous coronary intervention in complex coronary lesions: results of the CORA-PCI study (Complex Robotically Assisted Percutaneous Coronary Intervention). JACC Cardiovasc Interv. 2017;10(13):1320-1327.

8. Ghasem A, Sharma A, Greif DN, et al. The arrival of robotics in spine surgery: a review of the literature. Spine (Phila Pa 1976). 2018;43(23):1670-1677.

9. De Benedictis A, Trezza A, Carai A, et al. Robot-assisted procedures in pediatric neurosurgery. Neurosurg Focus. 2017;42(5):E7.

10. Gonen L, Chakravarthi SS, Monroy-Sosa A, et al. Initial experience with a robotically operated video optical telescopicmicroscope in cranial neurosurgery: feasibility, safety, and clinical applications. Neurosurg Focus. 2017;42(5):E9.

11. Bezerra HG, Mehanna E, W Vetrovec G, et al. Longitudinal geographic miss (LGM) in robotic assisted versus manual percutaneous coronary interventions. J Interv Cardiol. 2015; 28(5):449-455.

12. Hasan F, Bonatti J. Robotically assisted percutaneous coronary intervention: benefits to the patient and the cardiologist. Expert Rev Cardiovasc Ther. 2015;13(11):1165-1168.

13. Walters D, Omran J, Patel M, et al. Robotic-assisted percutaneous coronary intervention: concept, data, and clinical application. Interv Cardiol Clin. 2019;8(2):149-159.

14. Dangas G, Iakovou I, Nikolsky E, et al. Contrast-induced nephropathy after percutaneous coronary interventions in relation to chronic kidney disease and hemodynamic variables. Am J Cardiol. 2005;95(1):13-19.

15. Mahmud E, Schmid F, Kalmar P, et al. Feasibility and safety of robotic peripheral vascular interventions: results of the RAPID trial. JACC Cardiovasc Interv. 2016;9(19):20582064.

16. Smilowitz NR, Weisz G. Robotic-assisted angioplasty: current status and future possibilities. Curr Cardiol Rep. 2012; 14(5):642-646. 
17. Patel TM, Shah SC, Pancholy SB. Long distance tele-roboticassisted percutaneous coronary intervention: a report of firstin-human experience. EClinicalMedicine. 2019;14:53-58.

18. Madder RD, VanOosterhout S, Mulder A, et al. Feasibility of robotic telestenting over long geographic distances: a preclinical ex vivo and in vivo study. EuroIntervention. 2019; 15(6): e510-e512.

19. Albuquerque FC, Hirsch JA, Chen M, Fiorella D. Robotics in neurointervention: the promise and the reality. J Neurointerv Surg. 2020;12(4):333-334.

20. Plourde G, Pancholy SB, Nolan J, et al. Radiation exposure in relation to the arterial access site used for diagnostic coronary angiography and percutaneous coronary intervention: a systematic review and meta-analysis. Lancet. 2015; 386(10009):2192-2203.

21. Roguin A, Goldstein J, Bar O, Goldstein JA. Brain and neck tumors among physicians performing interventional procedures. Am J Cardiol. 2013;111(9):1368-1372.

22. Rajaraman P, Doody MM, Yu CL, et al. Cancer risks in U.S. radiologic technologists working with fluoroscopically guided interventional procedures, 1994-2008. AJR Am J Roentgenol. 2016;206(5):1101-1109.

23. Jacob S, Boveda S, Bar O, et al. Interventional cardiologists and risk of radiation-induced cataract: results of a French multicenter observational study. Int J Cardiol. 2013;167(5): $1843-1847$.

24. Andreassi MG, Piccaluga E, Gargani L, et al. Subclinical carotid atherosclerosis and early vascular aging from longterm low-dose ionizing radiation exposure: a genetic, telomere, and vascular ultrasound study in cardiac catheterization laboratory staff. JACC Cardiovasc Interv. 2015;8(4):616-627.

25. Reeves RR, Ang L, Bahadorani J, et al. Invasive cardiologists are exposed to greater left sided cranial radiation: the BRAIN study (Brain Radiation Exposure and Attenuation During Invasive Cardiology Procedures). JACC Cardiovasc Interv. 2015;8(9):1197-1206.

26. Klein LW, Tra Y, Garratt KN, et al. Occupational health hazards of interventional cardiologists in the current decade: results of the 2014 SCAI membership survey. Catheter Cardiovasc Interv. 2015;86(5):913-924.

27. Ross AM, Segal J, Borenstein D, et al. Prevalence of spinal disc disease among interventional cardiologists. Am J Cardiol. 1997;79(1):68-70.

28. Goldstein JA, Balter S, Cowley M, et al. Occupational hazards of interventional cardiologists: prevalence of orthopedic health problems in contemporary practice. Catheter Cardiovasc Interv. 2004;63(4):407-411.
29. Moore B, vanSonnenberg E, Casola G, Novelline RA. The relationship between back pain and lead apron use in radiologists. AJR Am J Roentgenol. 1992;158(1):191-193.

30. Britz GW, Panesar SS, Falb P, et al. Neuroendovascular-specific engineering modifications to the CorPath GRX Robotic System. J Neurosurg. Published online November 29, 2019. doi:10.3171/2019.9.JNS192113

31. Britz GW, Tomas J, Lumsden A. Feasibility of roboticassisted neurovascular interventions: initial experience in flow model and porcine model. Neurosurgery. 2020;86(2): 309-314.

32. Lendvay TS, Hannaford B, Satava RM. Future of robotic surgery. Cancer J. 2013;19(2):109-119.

33. Da L, Zhang D, Wang T. Overview of the vascular interventional robot. Int J Med Robot. 2008;4(4):289-294.

\section{Disclosures}

Dr. Jabbour: consultant for Medtronic and MicroVention. Drs. Tjoumakaris and Gooch: consultants for Stryker.

\section{Author Contributions}

Conception and design: Jabbour, Weinberg, Sweid. Acquisition of data: Weinberg, Sweid, Sajja. Analysis and interpretation of data: Jabbour, Weinberg, Sweid, Sajja. Drafting the article: Jabbour, Weinberg, Sweid. Critically revising the article: Jabbour, Weinberg, Sajja, Gooch, Herial, Tjoumakaris, Rosenwasser. Reviewed submitted version of manuscript: Jabbour, Weinberg, Sweid, Sajja. Approved the final version of the manuscript on behalf of all authors: Jabbour. Statistical analysis: Weinberg.

\section{Supplemental Information \\ Videos \\ Video 1. https://vimeo.com/424754254.}

\section{Correspondence}

Pascal Jabbour: Thomas Jefferson University Hospital, Philadelphia,PA.pascal.jabbour@jefferson.edu. 